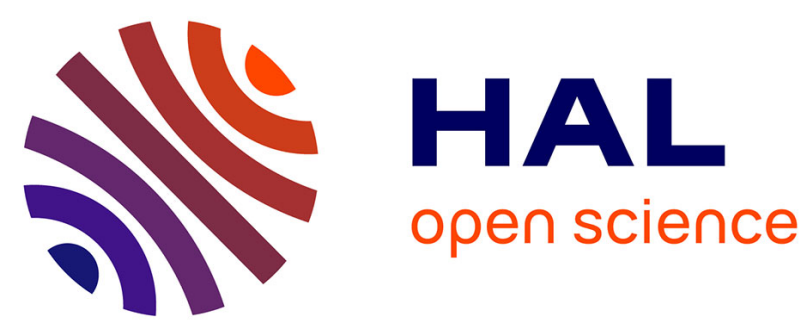

\title{
Rayleigh-Taylor Instability in Elastoplastic Solids: A Local Catastrophic Process
}

I Maimouni, Julie Goyon, E Lac, T Pringuey, J Boujlel, Xavier Chateau, Philippe Coussot

\section{To cite this version:}

I Maimouni, Julie Goyon, E Lac, T Pringuey, J Boujlel, et al.. Rayleigh-Taylor Instability in Elastoplastic Solids: A Local Catastrophic Process. Physical Review Letters, 2016, 10.1103/PhysRevLett.116.154502 . hal-01321013

\section{HAL Id: hal-01321013 https://hal-enpc.archives-ouvertes.fr/hal-01321013}

Submitted on 24 May 2016

HAL is a multi-disciplinary open access archive for the deposit and dissemination of scientific research documents, whether they are published or not. The documents may come from teaching and research institutions in France or abroad, or from public or private research centers.
L'archive ouverte pluridisciplinaire HAL, est destinée au dépôt et à la diffusion de documents scientifiques de niveau recherche, publiés ou non, émanant des établissements d'enseignement et de recherche français ou étrangers, des laboratoires publics ou privés. 


\title{
Rayleigh-Taylor Instability in Elastoplastic Solids: A Local Catastrophic Process
}

\author{
I. Maimouni, ${ }^{1,2}$ J. Goyon, ${ }^{1}$ E. Lac, ${ }^{2}$ T. Pringuey, ${ }^{2}$ J. Boujlel,${ }^{3}$ X. Chateau, ${ }^{1}$ and P. Coussot ${ }^{1}$ \\ ${ }^{1}$ Université Paris-Est, Laboratoire Navier (ENPC-IFSTTAR-CNRS), Champs sur Marne 77420, France \\ ${ }^{2}$ Schlumberger Riboud Product Center, Clamart 92140, France \\ ${ }^{3}$ IFPEN, Rueil-Malmaison 92500, France
}

(Received 11 November 2015; published 15 April 2016)

\begin{abstract}
We show that the Rayleigh-Taylor instability in elastoplastic solids takes the form of local perturbations penetrating the material independently of the interface size, in contrast with the theory for simple elastic materials. Then, even just beyond the stable domain, the instability abruptly develops as bursts rapidly moving through the other medium. We show that this is due to the resistance to penetration of a finger which is minimal for a specific finger size and drops to a much lower value beyond a small depth (a few millimeters).
\end{abstract}

DOI: 10.1103/PhysRevLett.116.154502

The Rayleigh-Taylor instability (RTI) is a well-known instability which occurs when a denser fluid rests on top of a lighter one [1]. As it develops, the two fluids penetrate one another, in the form of fingers. Instability is driven by the density difference and the acceleration to which the fluids are submitted, while surface tension provides a stabilizing effect. In contrast, RTI in solids is much less studied and understood, even though it relates to many application fields and can cause irreversible damage to structures. Examples include metal plates submitted to strong pressure or acceleration in high-energy density physics experiments [2], magnetic implosion of impactor liners [3,4], assessment of solid strength under high strain rate [5], slowly accreting neutron stars [6]. Other applications are found in geology: volcanic island formation [7], salt dome formation [8], and more generally, magmatic diapirism in Earth's mantle and continental crust $[9,10]$, correspond to situations where a liquid opens its way through a layer of denser solid material above it. In most approaches to this problem [7-9,11], the upper material was considered as a highly viscous fluid, which allowed simple simulations of the process, but could also be misleading. Another situation concerns oil well cementing operations, in which yield stress fluids of different densities (drilling muds and cement, e.g.), which behave as solids at rest, may be pumped into the well in an ill-favored density order [12].

The basic approach to RTI for solids assumes linear elastic materials. The problem appears similar to that for simple fluids, except that the role of surface tension effects, neglected for solids, is played by elasticity. For a single solid above a liquid with a (positive) density difference $\Delta \rho$, the instability criterion $(A)$ is given by $g \Delta \rho>4 \alpha \pi G / L$, where $G$ and $L$ are the shear modulus and length of the sample, respectively, and $g$ denotes the gravitational acceleration. Depending on boundary conditions, factor $\alpha$ was found to be $1[3,13], 1.6$ [14], or 2 [15]. A couple of experiments on metal plates [16] and with a yogurt [17] provided some support to this theory. From a more complete study [18] using soft elastic solids, the overall validity of this approach was proved but the wavelength was shown to be smaller than expected from theory and dependent on uncontrollable, slight disturbances of the surface [19].

RTI for solids is further complicated by the fact that yielding may occur beyond a critical deformation. So far, this aspect has been considered separately, leading to the conclusion that instability results from a sufficiently large initial perturbation amplitude $\varepsilon_{0}$ (penetration depth). The instability criterion $(B)$ then reads $g \Delta \rho>\beta \tau_{c} / \varepsilon_{0}$, where $\tau_{c}$ denotes the material's yield stress (in simple shear), and where $0.5 \leq \beta \leq 2$ depending on the sample aspect ratio $[13-15,18,24,25]$. Some tests with a single material were apparently in agreement with this criterion [17] but the plastic regime for this material was not so well-defined [19]. Finally, it was suggested [2] that elastic and plastic stability criteria should be taken into account successively, and deep theoretical analysis [26] predicted that for plastic materials, once the threshold is reached somewhere, the perturbation grows unlimitedly.

These approaches have the advantage of considering independently the elasticity and the yielding effects. However, one cannot exclude that the interplay of both mechanisms could play a crucial role in the early stage of the perturbation growth.

Here we aim at clarifying this problem through experiments on well-characterized materials, linearly elastic below a critical deformation and elastoplastic beyond this deformation. We show that the RTI in solids does not develop as predicted by the theory for simple elastic materials, but results from the ability of local perturbations to penetrate the material by involving, from the start, both elastic and plastic effects. At some point during the process, resistance to penetration drops, causing an abrupt 
instability in the form of bursts rapidly moving through the other medium.

Our RTI experiments are carried out in a rectangular tank (length $L=15 \mathrm{~cm}$, width $D=6 \mathrm{~cm}$, height $h=12 \mathrm{~cm}$ ) initially filled with a fluid of density $\rho$. A thin tab is placed in contact with the upper surface of this fluid, and a denser salt solution is $(0-10 \mathrm{~mol} / 1$ sodium iodide in water) slowly poured over it. Finally, the tab is withdrawn along the smallest side of the tank, and the interface is monitored with a 13 frame-per-second video device (see [19]). For Newtonian fluids as bottom and top media, we observe a transition to instability for $\Delta \rho / \rho$ in the range $0.4 \%-0.8 \%$. Considering the uncertainty on density measurements, the obtained values are in good agreement with the theoretical prediction in infinite space $\left(\Delta \rho / \rho=4 \pi^{2} \sigma / \rho g L^{2}=0.48 \%\right.$, where $\sigma=0.027 \mathrm{~N} \mathrm{~m}^{-1}$ ) for negligible edge effects [27], and approximating our system as a $2 \mathrm{D}$ one. As expected from theory, close to the critical $\Delta \rho$ value, the finger wavelength is of the order of the tank length [see Fig. 1(a)] and decreases for increasing $\Delta \rho$. Moreover, we checked
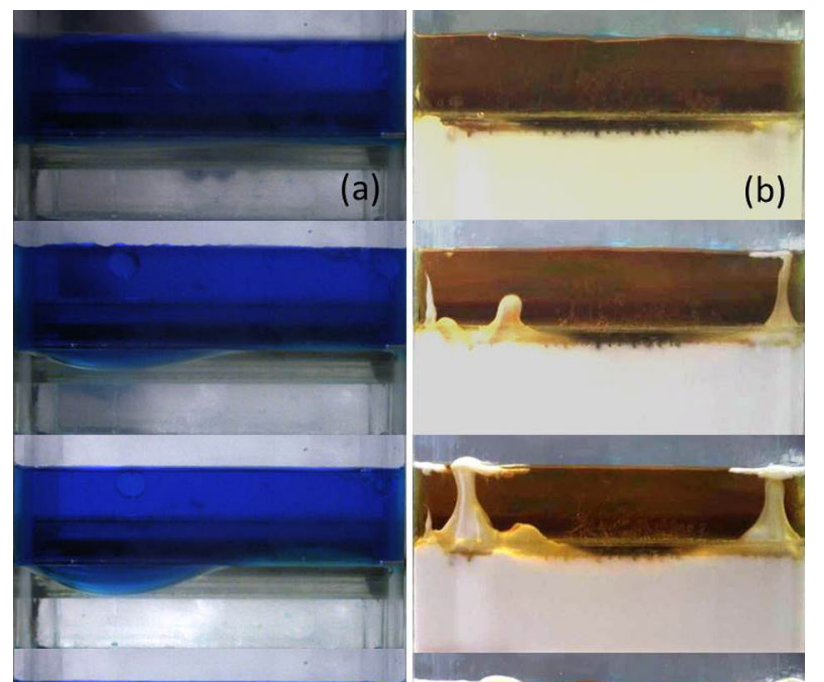

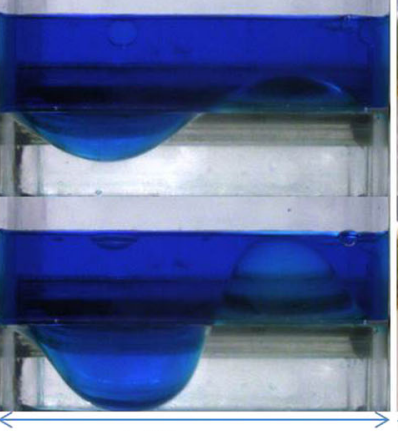

$L=15 \mathrm{~cm}$

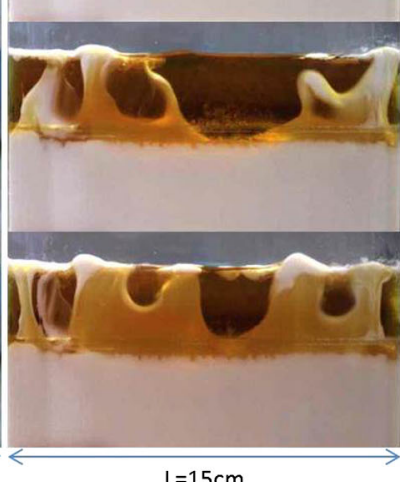

$\mathrm{L}=15 \mathrm{~cm}$
FIG. 1. RTI development for (a) viscous Newtonian fluid (Silicone oil, 0.35 Pa.s viscosity) initially below a denser colored water-ethanol solution $(\Delta \rho / \rho=1.3 \%$ ) at times (from top to bottom): 0 (midtime of gate opening), 6, 12, 18, and $24 \mathrm{~s}$; and (b) a concentrated (white) emulsion $\left(\tau_{c}=9.6 \mathrm{~Pa}\right)$ initially below a (brown) denser salt solution $\left(\Delta \rho=600 \mathrm{~kg} \mathrm{~m}^{-3}\right)$ at times: $0,1.9$, $3,3.8$, and $4.5 \mathrm{~s}$. that increasing the bottom fluid viscosity has no impact on the critical density difference and wavelength, other than simply making the whole fingering process slower.

We now turn to concentrated inverse emulsions as bottom material (see [19]). These emulsions are yield stress fluids (YSF); i.e., they behave as solids below a critical stress $\left(\tau_{c}\right)$ and flow like liquids beyond $\tau_{c}$ [28]. In the solid regime, they are purely elastic for sufficiently low stress, and elastoplastic at higher stresses as can be observed from the partial strain recovery for stress release (see Fig. 2). Two rheological parameters (same experimental conditions as in [29]), apparently related, (see inset of Fig. 2) well characterize the solid regime: the elastic modulus $G$ in the linear regime and the yield stress $\tau_{c}$, determined as the stress plateau from creep tests at a low, fixed shear rate.

In order to maintain the YSF in its solid state after it has been set up in the tank, we now gently place between its free surface and the tab a thin layer (about $3 \mathrm{~mm}$-thick) of salt solution with a density $20 \%$ smaller than that above the tab. When the tab is removed, the two salt solutions rapidly diffuse through each other, leading to a new top Newtonian fluid of density $\rho_{i}$. The tab displacement and this mixing induce negligible deformations of the YSF (see [19]). Besides, it was checked that the salt solution does not affect the emulsion behavior over the test duration.

Two very different situations are observed when the tab is withdrawn (in about $2 \mathrm{~s}$ ). In one case, the interface does not move at all, even after a long time (around one hour). In the other, despite the high apparent viscosity of the YSF, a very fast motion of the YSF into the Newtonian solution occurs in the form of 1 to $2 \mathrm{~cm}$-wide mushroomlike bursts. These bursts penetrate the liquid above at different distances from the walls (as seen from their different colors in Fig. 1) and reach the top of the tank in a time of the order of

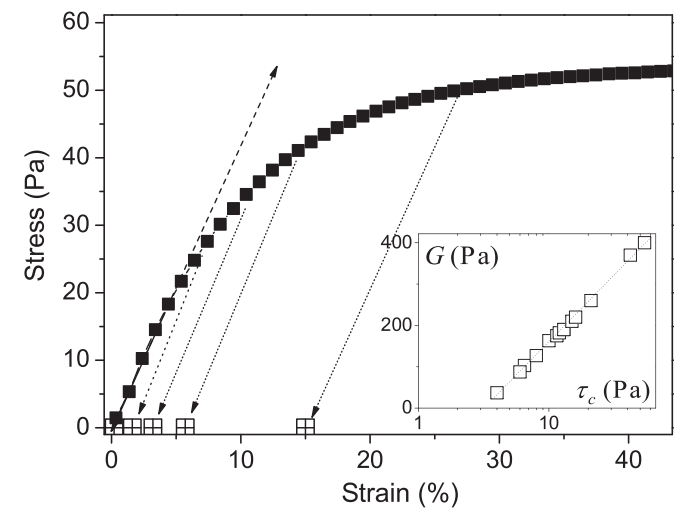

FIG. 2. Creep test for an emulsion $\left(\tau_{c}=54 \mathrm{~Pa}\right)$ : (filled squares) stress vs strain when applying a stress ramp; (crossed squares) final strain after stress release (no stress imposed) at some point during the ramp; dotted lines, stress-strain path. Inset: $G$ measured in the region of negligible final strain after stress release, as a function of yield stress, for emulsions at different concentrations; the dotted line corresponds to $G=140 \ln \left(\tau_{c} / 3.2\right)$. 
one second. Soon after this stage, new fingers appear, and eventually, the two fluids fully overlap. Although not visible in Fig. 1, Newtonian fluid fingers form and penetrate the YSF in a similar way to YSF fingers penetrating the Newtonian fluid but in the opposite direction.

Additional features are remarkable: (i) in unstable cases, even under conditions close to stability, the instability starts soon after the beginning of tab withdrawal; (ii) the transition to instability occurs under almost the same conditions when the tank length is 7 or $15 \mathrm{~cm}$ (see Fig. 3); (iii) in our range of experimental conditions (tank size, yield stress), the unstable bursts size (i.e., half the instability wavelength) approximately keeps a constant value, significantly smaller than the interface length.

Thus, the RTI for YSF exhibits striking differences with the instability for simple fluids: just at the transition, which is independent of the sample length, fingers, much smaller than the container, form and develop in a catastrophic way through a material of relatively high apparent viscosity. This demonstrates that the elastic theory, which predicts qualitative trends akin to those for simple fluids at the instability onset, does not apply.

The transition between stable and unstable situations occurs when either $\Delta \rho=\rho_{i}-\rho$ is increased, or when $G$ (or equivalently, $\tau_{c}$ ) is decreased (see Fig. 3 ). These results are reproducible with an uncertainty of $10 \%$ on the critical elastic modulus value. It appears that the stable and the unstable cases are approximately separated by a line going through the origin, $G \propto \Delta \rho$ (see Fig. 3). This result cannot be considered as corresponding to criterion $(A)$ for the above reasons. Alternatively, the instability could be governed by the yielding of the fluid for sufficient initial perturbation amplitudes, which would lead to a criterion akin to $(B)$ corresponding to $\tau_{c} \propto \Delta \rho$, which can also

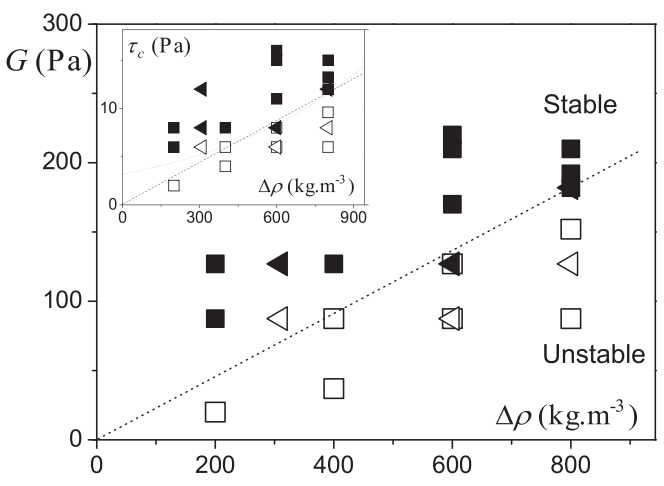

FIG. 3. RTI results for YSF in terms of elastic modulus as a function of density difference for $L=7 \mathrm{~cm}$ (triangles) or $L=15 \mathrm{~cm}$ (squares): stable cases (filled symbols), unstable cases (open symbols). The dotted line is a guide for the eyes. The inset shows the same data represented in terms of $\tau_{c}$ with the corresponding dotted line (consistent with the $G$ vs $\tau_{c}$ variation) and a straight dashed line as a guide for the eye. roughly represent the transition (see inset of Fig. 3). With such solid-liquid materials, it is difficult to get a perfectly planar initial free surface, there indeed always remains some unevenness, in particular, along the corners of the interface [see Fig. 1(b)]. This criterion (with $\beta=1$ ) would effectively predict our instability limit if the YSF upper surface unevenness had a constant amplitude under all experimental conditions $\left(\varepsilon_{0}=1.4 \mathrm{~mm}\right)$, which is not realistic. Moreover, a stable situation close to the instability limit remains stable unless we strongly perturb the interface, for example, by digging a finger-size object over about two centimeters into the YSF. This confirms that the instability does not simply develop as a result of significant initial perturbation amplitude.

These observations demonstrate that the initial perturbation growth, leading to fingering, differs from usual instability processes considered so far. More precisely, they suggest that, under some conditions, the fingering phenomenon has a more local origin than expected. In order to further investigate the process of finger growth, we measure the needed force to push an object (thickness $e$, width $7 \mathrm{~cm}$ ) with a rounded edge (radius of curvature $e / 2$ ) vertically against the interface at the center of an emulsion bath in a cylindrical container (13 cm diameter). We compute the resisting force $(F)$ by withdrawing the buoyancy force, estimated assuming an exact immersion of $h$ and taking into account the rounded shape of the object edge. Note that similar results are obtained with different types of plate surface (rough or smooth and hydrophilic or hydrophobic) (see [19]), which means that the plate-fluid interface plays a minor role here. This supports the idea that this test mimics the penetration of a fluid finger through the YSF with negligible viscous stress resulting from the contact between the two fluids.

The first critical observation is that, for a YSF, $F$ grows much less rapidly with $h$ than for a linear elastic material (a gelatin) of a similar elastic modulus (see Fig. 4), even for very low depth, i.e., corresponding to very small deformation. This means that some plastic flow occurs as soon as the penetration starts. Actually, if we inverse the motion direction from some depth, the deformation recovery is very small even for small $h$ (i.e., small deformation) (see Fig. 4). This indicates that, in contrast with creep tests in simple shear, (see Fig. 2) the penetration is mostly related to plastic flow from the beginning of the process.

It is likely that the interface deformation, even when small, immediately induces plastic deformations around the plate but the rest of the material undergoes elastic deformations. This is consistent with the observation that, during a reverse motion of the plate, the force curve decreases towards zero with a slope similar to that of the force vs depth curve for gelatin of same elastic modulus (see Fig. 4). This shows that most of the material is deformed elastically as for a pure elastic solid, but the force needed for penetration is much lower due to a plastic flow in a small 


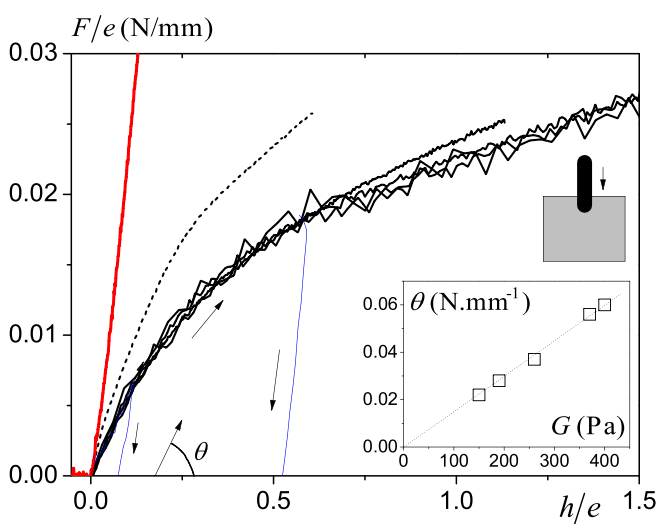

FIG. 4. Plate pushed against a YSF $\left(\tau_{c}=54 \mathrm{~Pa}\right)$ bath at a velocity of $0.3 \mathrm{~mm} / \mathrm{s}$ : force as a function of penetration depth, both rescaled by the plate thickness for different $e$ (from bottom to top): 2, 4, 8, $16 \mathrm{~mm}$ (black continuous lines), $30 \mathrm{~mm}$ (dashed line). Thick (red) continuous line: simple elastic material (gelatin, $G=400 \mathrm{~Pa}$ ) with $e=8 \mathrm{~mm}$. Thin (blue) continuous lines: recovery tests. The arrows show the direction of plate motion. Inset: initial slope of the curve $(\theta)$ as a function of $G$ (for the same emulsion at different concentrations).

region around the plate, and thus, the deformations induced in the rest of the material are smaller.

The $F(h)$ curves exhibit simple generic trends. In a first stage, i.e., up to $h \approx e / 2, F$ is independent of speed (in the range $0.01-3 \mathrm{~mm} / \mathrm{s}$ ) and of $e$ as long as $e / L$ is sufficiently small, so that we obtain a master curve when rescaling $F$ and $h$ by $e$ (see Fig. 4). These results mean that, in the initial regime, the penetration process essentially involves (elastic and plastic) deformations in the solid state and develops similarly at the different scales as long as we are far from the edges. Moreover, similar tests with the same material type at different concentrations show that $F^{\prime}(h)_{h \rightarrow 0}=\theta$ is simply proportional to $G$ (see Fig. 4, inset).

Turning back to the RTI problem, we deduce that the force (per unit length of plate in the horizontal direction) associated with the beginning of a perturbation's penetration may be approximately written $\chi G h$, where $\chi$ is constant for a sufficiently small perturbation wavelength to container size ratio and increases beyond some critical value $e_{c}$ (see Fig. 4) (here, $e_{c} / L=0.15 \pm 15 \%$ ). Note that $\chi$ depends on shape of the perturbation and $e_{c}$ depends on the size of the container. Thus, we can expect the situation to be unstable if a perturbation of small size can overcome the resisting force in the first regime. Some irregularities of the interface due to its unevenness lead to the existence of preexisting perturbations of various shapes. Let us consider one of them of width $e$; the additional force (per unit length) resulting from density difference, if the perturbation digs further of a (small) depth $h$ while keeping its front shape, is $\Delta \rho g e h$. As a consequence, as long as $\chi G>\Delta \rho g e$, the situation is stable. Otherwise, if $\chi G<\Delta \rho g e$, any slight additional digging of a small $h$ value will degenerate, an instability will occur. Since $\chi$ increases faster than $e$ for $e>e_{c}$, as appears in Fig. 4 (see force curve for $e=30 \mathrm{~mm}$ ), the first unstable case reached when increasing $\Delta \rho$ or decreasing $G$ corresponds to $e=e_{c}$. Finally, assuming that all the perturbation sizes are equally probable, we get a criterion of instability formally analogous to (A), but (i) with an origin of instability which is now essentially local, meaning here sufficiently far from the boundaries, and (ii) with a major role of plastic flow. It is remarkable that the typical finger width value expected from the above approach $\left(e_{c} \approx 2 \mathrm{~cm}\right)$ is close to that observed in our RTI (in a different geometry). These results also imply that surface tension effects do not play any role in this instability in our range of material properties (see [19]).

Another specific feature of RTI with yield stress fluids is the rapidity at which the perturbation progresses even very close to the instability limit. The origin of this catastrophic process can be identified through the $F(h)$ curves. Indeed, between $h=e / 4$ to $h=e$, the slope $\left[F^{\prime}(h)\right]$ of the master curve strongly decreases (by a factor of about 9), such that the ratio of $F$ to $\Delta \rho g e h$ drops dramatically as the perturbation further progresses.

This behavior can be explained by the observation of the flow field surrounding the plate. It was recently shown from direct velocity field measurements inside the fluid $[29,30]$ that, in this regime, a liquid region of uniform thickness $(\lambda)$ develops along the plate, where the fluid is almost homogeneously sheared (at a shear rate $\dot{\gamma} \approx V / \lambda$ ) while the rest of the material (outside this uniform layer) is only deformed in its solid regime as the plate advances. In that case, the resisting force per unit length is roughly $2 \tau(\dot{\gamma}) h$ (neglecting the edges), which decreases to $2 \tau_{c} h$ as $V \rightarrow 0$. In addition, as from our data $\chi \approx 2$ and $\tau_{c}<0.15 G$ (see Fig. 2, inset) in our range of tests, we deduce that $\chi G \gg \tau_{c}$. This inequality is even stronger for a liquid finger penetrating a YSF as the stress along the vertical interface is certainly lower. Thus, as the initiation of the instability requires $\Delta \rho g e>\chi G$, once this regime is reached, the force due to density difference rapidly becomes much larger than the resisting force allowing a much faster motion of the perturbation. This explains the catastrophic effect observed in practice, which contrasts with the long-term stability obtained for slightly different conditions in terms of $\Delta \rho$ or $\tau_{c}$.

The above description is supported by a different set of experiments in which the elastoplastic regime is bypassed. This is performed by setting the material in its liquid regime just before allowing any contact between the two fluids. To this aim, we remove the intermediate liquid layer so that the tab is now in contact with the YSF. It appears that $G$ is not the relevant parameter in this case: even for very low $\Delta \rho$, the instability occurs for high values of $G$ (see [19]), although the stability limit can still be approximated by a model of the form $\tau_{c} \propto \Delta \rho$. As the tab is removed, it shears 
the upper material layers, which then reach the liquid regime. Then, the perturbation no longer needs to overcome the elastoplastic resistance and progresses immediately through the YSF.

The description of the Rayleigh-Taylor instability for elastoplastic material, i.e., nonpurely elastic solid materials, requires a change of paradigm: the boundary conditions are not as important as for simple solid or liquid materials; the instability is basically a local, catastrophic process.

[1] S. J. W. Rayleigh, Proc. London Math. Soc. s1-14, 170 (1882); D. H. Sharp, Physica (Amsterdam) 12D, 3 (1984).

[2] A. R. Piriz, Y. B. Sun, and N. A. Tahir, Phys. Rev. E 88, 023026 (2013).

[3] G. Terrones, Phys. Rev. E 71, 036306 (2005).

[4] J. J. López Cela, A. R. Piriz, M. Temporal, N. A. Tahir, and M. C. Serna Moreno, Eur. Phys. J. Appl. Phys. 29, 247 (2005).

[5] R. T. Olson, E. K. Cerreta, C. Morris, A. M. Montoya, F. G. Mariam, A. Saunders, R. S. King, E. N. Brown, G. T. Gray, and J. F. Bingert, J. Phys. Conf. Ser. 500, 112048 (2014); D. B. Sinars et al., Phys. Plasmas 18, 056301 (2011).

[6] O. Blaes, R. Blandford, and P. Madau, Astrophys. J. 363, 612 (1990).

[7] B. D. Marsh, J. Geol. 87, 687 (1979).

[8] S. Zaleski and P. Julien, Tectonophysics 206, 55 (1992).

[9] D. Bittner and H. Schmeling, Geophys. J. Int. 123, 59 (1995).

[10] E. B. Burov and P. Molnar, Earth Planet. Sci. Lett. 275, 370 (2008); W. Gorczyk and K. Vogt, Gondwana Res. 27, 196 (2015).

[11] P. Molnar and G. A. Houseman, Geophys. J. Int. 158, 1134 (2004); G. A. Houseman and P. Molnar, Geophys. J. Int. 128, 125 (1997).

[12] S. Bittleston and D. Guillot, Oilfield Rev.3, 44 (1991).

[13] A. R. Piriz, J. J. López Cela, O. D. Cortázar, N. A. Tahir, and D. H. H. Hoffmann, Phys. Rev. E 72, 056313 (2005).

[14] J. W. Miles, General Dynamics Reports No. GAMD-7335 and No. AD 643161 (1966) (unpublished).

[15] A. C. Robinson and J. W. Swegle, J. Appl. Phys. 66, 2859 (1989).

[16] J. F. Barnes, P. J. Blewett, R. G. McQueen, K. A. Meyer, and D. Venable, J. Appl. Phys. 45, 727 (1974); J. F. Barnes,
D. H. Janney, R. K. London, K. A. Meyer, and D. H. Sharp, J. Appl. Phys. 51, 4678 (1980).

[17] G. Dimonte, R. Gore, and M. Schneider, Phys. Rev. Lett. 80, 1212 (1998).

[18] S. Mora, T. Phou, J. M. Fromental, and Y. Pomeau, Phys. Rev. Lett. 113, 178301 (2014).

[19] See Supplemental Material at http://link.aps.org/ supplemental/10.1103/PhysRevLett.116.154502 for previous experiments with soft materials-Material propertiesScheme of the experimental set up-Timing of tab withdrawal and impact on fluid flow-Surface tension effects-Results for a direct contact between the tab and the YSF during tab removal-Plate penetration in a YSF, which includes Refs. [20-23].

[20] P. Coussot and F. Gaulard, Phys. Rev. E 72, 031409 (2005).

[21] P. Coussot, Q. D. Nguyen, H. T. Huynh, and D. Bonn, J. Rheol. 46, 573 (2002); Phys. Rev. Lett. 88, 175501 (2002); P. Coussot, J. S. Raynaud, F. Bertrand, P. Moucheront, J. P. Guilbaud, H. T. Huynh, S. Jarny, and D. Lesueur, Phys. Rev. Lett. 88, 218301 (2002); J. S. Raynaud, P. Moucheront, J. C. Baudez, F. Bertrand, J. P. Guilbaud, and P. Coussot, J. Rheol. 46, 709 (2002); G. Ovarlez, S. Rodts, X. Chateau, and P. Coussot, Rheol. Acta 48, 831 (2009).

[22] T. Chevalier, S. Rodts, X. Chateau, J. Boujlel, M. Maillard, and P. Coussot, Europhys. Lett. 102, 48002 (2013).

[23] P. Coussot, H. Tabuteau, X. Chateau, L. Tocquer, and G. Ovarlez, J. Rheol. 50, 975 (2006).

[24] D. C. Drucker, Ingenieur Archiv. 49, 361 (1980).

[25] A. R. Piriz, J. J. López Cela, and N. A. Tahir, J. Phys. Conf. Series 244, 022007 (2010).

[26] S. M. Bakhrakh, O. B. Drennov, N. P. Kovalev, A. I. Lebedev, E. E. Meshkov, A. L. Mikhailov, N. V. Nevmerzhitsky, P. N. Nizovtsev, V. A. Rayevsky, G. P. Simonov, V. P. Solovyev, and I. G. Zhidov, Hydrodynamic Instability in Strong Media, LLNL Report No. UCRL-CR-126710.

[27] F. Charru, Hydrodynamic instabilities (Cambridge Texts in Applied Mathematics, Cambridge, 2011).

[28] T. G. Mason, Current Opinion in Colloid \& Interface Science, 4, 231 (1999).

[29] J. Boujlel, M. Maillard, A. Lindner, G. Ovarlez, X. Chateau, and P. Coussot, J. Rheol. 56, 1083 (2012).

[30] M. Maillard, J. Boujlel, and P. Coussot, Phys. Rev. Lett. 112, 068304 (2014); J. Non-Newtonian Fluid Mech. 220, 33 (2015). 